\title{
Business Analytics Capabilities for Organisational Resilience
}

\author{
Hussein Al-Ghattas \\ University of Technology Sydney \\ Hussein.f.al-ghattas@student.uts.edu.au
}

\author{
Olivera Marjanovic \\ University of Technology Sydney \\ Olivera.Marjanovic@uts.edu.au
}

\begin{abstract}
Nowadays, organizations are facing unique challenges created by different disruptions, including natural disasters, new technologies, regulatory changes, and more recently, a global pandemic. Consequently, the need to build, sustain, and continuously enhance Organizational Resilience (OR) is greater than ever. An ongoing process of building $O R$ requires high-quality data and business analytics (BA) capabilities. In this paper we aim to investigate the yet-to-be explored link between $B A$ and OR. We achieve this aim by conducting a multidisciplinary literature review on $O R$ and $B A$, focusing on BA capabilities for OR. Based on our findings, we then propose a conceptual framework of $B A$ capabilities for $O R$. In doing so, we also bring a well-established area of $O R$ to the attention of $B A$ researchers, as a critically important area for further $B A$ research and practice.
\end{abstract}

\section{Introduction}

Organisations of all types are continuously facing different types of disrupting events, with some even threatening their very existence [1, 2]. Industry reports offer some interesting insights about companies' experiences with major disruptions. For example, according to a survey about corporate crisis by PWC, $42 \%$ of 1,400 participating organisations, all with prior experience with major crisis, indicated that they were in a better position post-crisis, with some of them even reporting revenue growth [1]. Another industry report by McKinsey, which traced the performance of more than 1,000 publicly traded companies operating across multiple industries and geographies during the global financial crisis (20072011), reported that 10 percent of these companies outperformed their counterparts [3].

Therefore, it is not surprising that the phenomenon of organisational resilience (OR) continues to attract researchers' attention, now for many years, and predominantly in the business discipline. However, as Heeks and Ospina [4] observes, there is limited research on the potential role of information systems (IS) in enhancing OR.

OR is also dependent on a set of capabilities, including cognitive, behavioural, emotional, and relational capabilities [5]. Organisations need to develop these capabilities across all phases of OR, which include anticipation, coping and adaptation [6]. For example, during the anticipation phase of OR, cognitive capabilities such as observation and identification are needed to anticipate and detect signs of future threats and major change events [7]. In other phases of OR, the same capabilities will manifest in different ways.

Furthermore, an ongoing process of building and sustaining OR requires high-quality data, and nowadays Business Analytics (BA). For example, in response to being severely disrupted by fintech, member-owned Credit Unions turned their attention to data and analytics, looking for new ways to serve their members [8]. In another example a retailer, disrupted by the current pandemic, used advanced analytics to reduce its range of products, improve efficiency, reduce its procurement costs, and implement a new operating model [9].

This research aims to investigate the role of Business Analytics (BA) in OR. In particular, we focus on the role and Business Analytics (BA) capabilities in improving OR. Following [10], we define (BA) capabilities "as the ability of the firm to capture and analyse data towards the generation of insights, by effectively deploying its data, technology and talent through firm-wide processes, roles and structures", p.274 We view OR as a multi-faceted phenomenon, defined as “an organisation's ability to anticipate potential threats, to cope effectively with adverse events, and to adapt to changing conditions" [6], p.220.

Against this background, our broader research project focuses on the role of BA capabilities in building and sustaining organisational resilience in 
different organisational contexts. In this paper we aim to explore the following main research question:

What does the current multidisciplinary literature say about the role of $B A$, in particular $B A$ capabilities in building and sustaining OR?

We answer this question by conducting a structured review of the multidisciplinary literature published in both OR and BA, focusing on capabilities. Our findings confirm that BA and OR continue to be investigated in disciplinary silos, with very limited number of papers focusing on both. Consequently, we argue for a more synergy across the two fields.

On the OR side, we confirm the previous observation by Heeks and Ospina [4]about the need to bring the IS perspective to the OR research. We argue that this perspective should include BA.

Similarly, on the BA side we find a lack of research on OR. Here we propose to extend the current research on business value of BA to include OR. In particular, we observe the need to investigate how BA capabilities lead to $\mathrm{OR}$ in different organisational contexts.

Based on our insights from the multidisciplinary literature review, we propose a conceptual framework describing BA capabilities for OR. We use it to articulate a number of interesting future research questions for BA researchers.

The structure of the remainder of this paper is as follows. The next section provides foundation concepts used in this research. Section 3 offers a step by step detail of the literature review research method, followed by Section 4 which describes our research findings. Section 5 describes the proposed theoretical framework. Finally Section 6 offers some interesting questions for future BA research, describes the main limitations of this research study and our future work.

\section{Foundation Concepts}

\subsection{Organisational Resilience}

The topic of resilience has been studied in a number of research disciplines including psychology, engineering, ecology, environmental science, organisation science, business and management [5, 11]. Across all disciplines the concept of resilience is indicative of strength, dedication and practical awareness of, and responsiveness to disruptive events, as well as robustness during situations of stress and change [12] [11]. In this research we consider the concept of resilience form the organisational perspective. This perspective is different from the so-called individual (i.e. personal) resilience that is commonly understood as a personal characteristic of an individual.

Conceptualisation of resilience in the business and management research implies multiple themes, all focused on different capacities and abilities of an organisation. For some scholars OR is understood as a recovery capacity back to a (new) normal state post-major crisis, others include the capacity to improve organisational process and capabilities, while the third group adds the anticipation capacity in their understanding of resilience [6].

We view OR as a multi-faceted phenomenon, defined as "an organisation's ability to anticipate potential threats, to cope effectively with adverse events, and to adapt to changing conditions" [6], p. 220 .

While earlier research had treated resilience as an event or the end-state [13], more recent studies emphasise the resilience as a process rather than an one-off reaction [5]. Consequently, there is a need to better understand different actions taken over time, which result in a more resilient organisation [14] [15] [5]. The process perspective of resilience is also indicative of "the dynamic nature of resilience as an interaction between the organisation and the environment" [5], p.742

Following Duchek [6] in this paper we conceptualise OR as a process that includes three consecutive stages: anticipation, coping and adaptation. The process perspective also "links organisational capabilities to outcomes" [16]. A conceptual model of OR, previously proposed by Duchek [6] and depicted by Figure 1, shows different stages of an OR process, along with corresponding organizational capabilities. We use this conceptual model to ground our exploration of BA capabilities for OR.

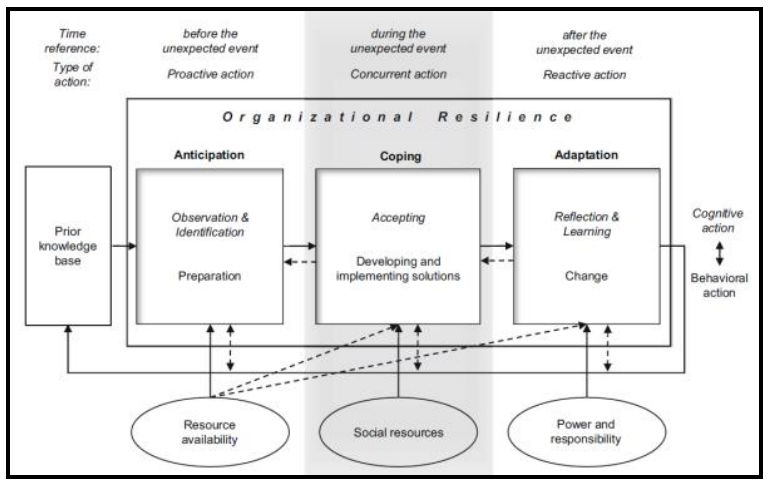

Figure 1: Duchek's conceptual framework of OR[6] 


\subsection{Business Analytics (BA) and BA Capabilities}

Business analytics (BA), also known as Business Intelligence and Analytics (BI\&A) [17], continues to attract the attention of both academic and business communities, now for decades [18]. The origins of BA could be traced back to the early 1970 s and the long-established area of Decision Support Systems (DSS) that later evolved into Business Intelligence (BI) in the 1990s, and BA in 2000s, which was recently expanded to include big data [19].

A very dynamic nature of this discipline has led to multiple definitions and terms among scholars and practitioners, often from different disciplines. While for example [18] and [20] used the term "Business intelligence and analytics", the emergence of big data have prompted the researchers to use the term "Big Data Analytics" [10, 17, 21]. Such inconsistency of interpretation of the foundation concepts in this discipline has resulted in the exiting "conceptual confusion", as noted by [22].

In this paper we adapt the term Business Analytics (BA) as a holistic, all-encompassing term, based on Chen et al.'s (2012) [18] definition of BI\&A. Understood in this way, BA includes technical infrastructure for storage, management and processing of different types of data (including big data), various BI/BA applications, descriptive, prescriptive and predictive analytics models and methods, as well as the related organisation practices such as data strategy, data governance and, most importantly for this research BA capabilities [23].

The concept of BA capabilities originates from an earlier conceptualization of IT capabilities. For example, Aral et al. [24] defined IT capabilities as "interlocking systems of practices and competencies that complement IT", p.765 Based on their empirical research, Aral et al.'s model highlighted the positive impact of the combined IT assets and IT capabilities on the organisations performance, market value and innovation [24]

BA Capabilities, defined earlier in the paper, include decision makers' capability to interpret organisational data and take value-adding actions, which in turn may lead to improved business processes, enhanced decision making, and different forms of business value [25].

Other researchers consider BA capabilities to include customer relations capabilities, which refer to competences and practices developed through the use of BA tools to build insights and make decisions in the context of customer-facing processes[26]
While they may be defined in different ways, BA capabilities emphasize the value created by interpreting data, and turning insights into valueadding actions. In this research, we are particularly interested in BA capabilities that could lead to a more sustainable OR.

\section{Research Approach - Structured Literature Review}

Following and combining the literature review methods by Webster and Watson [27] and Vom Brocke et al. [28], our literature review included the following five steps: 1) Definition of the review scope; 2) Identification of information sources; 3) Search process; 4) Literature analysis \& synthesis; and 5) Direction for future research. These steps that are described as follows.

\section{Step 1: Definition of review scope}

Our multidisciplinary literature review focused on two different areas of research: organisational resilience (OR) and BA capabilities. In the OR field we were particularly interested in finding any previous research on data, analytics and organizational capabilities, which included those related to analytics. In the BA field we focused on BA capabilities in general, looking for prior research where these capabilities were related to OR in any way. Looking across OR and BA fields we were particularly interested in any prior research that combined the two fields. Figure 2 illustrates the scope of our literature review.

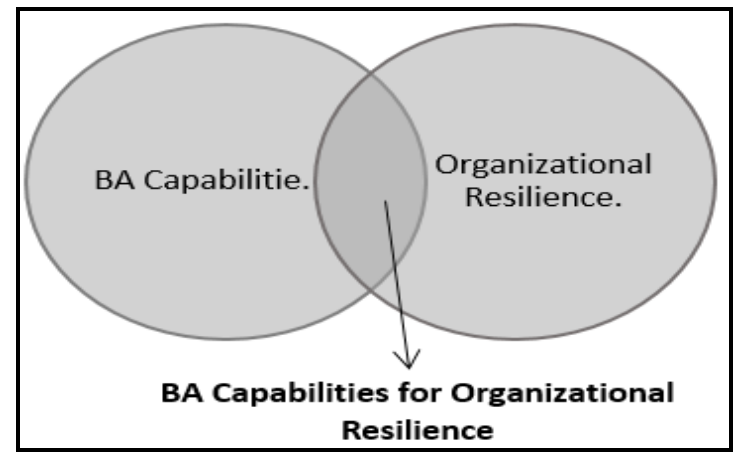

Figure 2. The scope of our literature review.

\section{Step 2: Identification of information sources}

We identified Web of Science, Scopus, Science Direct, Emerald insights, ABI/INFORM, EBSCO, JSTOR, and Google Scholar as suitable sources of OR literature. We also searched the Association of 
Information Systems (AIS) electronic library to look for any research on OR.

To explore BA capabilities reported in previous literature, we needed to perform a broader BA-related search within IS journals and conferences including:

- Proceedings of leading IS-related conferences including AMCIS, HICSS, ACIS, PACIS, ICIS, and ECIS.

- AIS electronic library, which included the leading IS journals

- Journal of Business Research, and

- European Journal of Operational Research.

We also performed forwards and backwards search using Google Scholar to look for additional sources, including academic journals and industry reports.

\section{Step 3: Search process}

In this step we proceeded with the selection of the most relevant keywords to guide our search process in both OR and BA fields. The following keywords combinations were defined to perform the searches across all databases: ("organi?ational resilience" OR "business resilience" OR "management resilience" OR "corporate resilience" OR "enterprise resilience" OR "industry resilience" OR "resilient organi?ation")

We then used the following keyword combination to search above sources: (("Big data analytics" or "Business Intelligence" or "Business Analytics" or "BA" or "business intelligence \& analytics" or "organizational analytics" or "Analytics" or "business analytics systems") AND capabilit*).

Searching other databases listed above for ORrelated articles resulted in a total of 5752 articles with 2147 articles from the Web of Science, 400 articles from Science Direct, 881 articles from Scopus, 243 articles from Emerald insight, 767 articles from ABI/Inform, 792 from EBSCOhost, 11 articles from JSTOR and 269 articles from Taylor \& Francis. After reviewing of title and abstract of these papers, we observed an existing fragmentation of the definition and conceptualisation of OR. Most importantly we identified the OR literature review and other agenda setting papers that are well cited within the area of OR

Consequently, we turned our attention to these publications - see [5] [29] and [30]. Through forwards and backwards search, these three influential publications enabled us to collect additional OR papers. Guided by these publications, we were also able to get important insights into the current trends in OR research [29], a detailed categorisation of perspectives, concepts and methodologies within OR literature [30], and a process view OR [5], which is particularly relevant for our research. When reviewing OR literature, we looked for any conceptualisation of OR-related capabilities, looking for those that could be related to data and analytics.

Given our disciplinary focus on IS, we also searched the IS sources (i.e. the basket of 8) using the OR-related keywords. This particular search resulted in a total of 35 publications, as shown by Table 1 .

We then turned our attention to BA, in particular BA capabilities, and performed search using the previously described method. This resulted in a total of 164 publications, published between 1999 and today. Only 81 publications were directly evaluating the value of BA capabilities for organizational level outcomes. We judged those to be relevant for our research, as $\mathrm{OR}$ is related to the outcomes at the organizational level.

Another 29 records addressed the how and what contributes to development of BA capabilities and two papers presented a maturity models of BA capabilities

\begin{tabular}{|l|c|}
\hline \multicolumn{1}{|c|}{ Journal } & Results \\
\hline European Journal of Information Systems & 4 \\
\hline Information Systems Journal & 1 \\
\hline Information Systems Research & 2 \\
\hline Journal of Information Technology & 6 \\
\hline Journal of Strategic Information Systems & 7 \\
\hline MIS Quarterly & 3 \\
\hline Journal of AIS & 5 \\
\hline Journal of MIS & 7 \\
\hline
\end{tabular}

Table 1. OR-related papers in the basket of 8 IS journals.

Step 4: Literature analysis \& synthesis

Conceptualising organisational resilience as an ongoing process enabled us to frame and organise our research findings to correspond to different phases of the process. Our analysis and synthesis resulted in the key findings described in the following section.

\section{Step 5: Directions for Future Research}

Based on our findings, we then proceed to articulate some interesting directions for future research. They are described in the concluding section of this paper. While we see opportunities for multidisciplinary research, in this paper we focus on BA-related areas of future research, which are opened up by OR. 


\section{Research Findings}

In answering our research question: What does the current multidisciplinary literature say about the role of BA, in particular BA capabilities in building and sustaining OR? we offer the following key findings.

Both OR and BA research are conducted within their respective disciplinary silos. We found only one BA research publication [31] that is explicitly dealing with BA for OR. We argue that the increased uncertainly and complexity of today's business environments present an opportunity for both OR and BA researchers to combine their efforts and well-developed disciplinary bodies of knowledge, in order to enable more resilient organisations.

Our research findings also confirm the previous finding by [4], that the IS/IT perspective is still missing from the current OR literature. More specifically, we did not find any OR focused research that considered data and analytics, let alone BA capabilities. This is an important research gap in the OR literature as well as an opportunity for BA researchers to engage with a well-established OR community.

On the other side, we found that the BA field (broadly defined), continues to focus on business value creation and competitive advantage [32]. However, there is a clear knowledge gap in BA research related to OR. We could only find a single study that used quantitative research method to explore the relationship between what they refer to as organizational analytical capabilities (OAC), organizational resilience (OR) and the business process management maturity (BPMM) [31]. We expect more OR-related research in BA, which is promoted by the current pandemic.

\section{Towards a Research Framework of BA Capabilities for OR}

Based on these research gaps discovered in both $\mathrm{BA}$ and OR, we observed the need for a new framework that could combine insights from these two fields. Based on the literature analysis and synthesis, we identified the need to identify and combine: (1) Organisational resources (2) BA capabilities; and (3) Organizational resilience capabilities. Consequently, we propose such a framework (Figure 3) by combining insights from prior literature from both disciplines $\mathrm{OR}$ and $\mathrm{BA}$ capabilities, as follows.
To conceptualize the first component of our proposed framework, Organizational resources, we adopt Gupta and George's[17] categories of organisational resources namely, tangible, human skills and intangible resources. According to Gupta and George, these resources are required for development of a robust set of BA capabilities. As they explain, tangible resources are the one which can be exchanged in the market like physical assets, technology and data. Intangible resources include, for example, data driven culture and knowledge. The third category of resources, human skills, include leadership and other technical skills[18]. We posit that these categories need to be reconceptualised and possibly extended to include intangible, tangible and human skills resources across different stages of the OR process, as identified by Duchak [6].

For the second component of our framework, BA capabilities, we propose to extend the Business Analytics Capability Framework (BACF) by [33] to include OR-related capabilities. The BACF framework classifies BA capabilities into four main areas: (1) Governance capability area, (2) Culture capability area, (3) Technology capability area and (4) People capability area. The framework presents a comprehensive categorisation for areas that contributes to the build of BA capabilities. We posit that the same BA capability areas are also highly relevant for OR.

To conceptualise the third component of our proposed framework, Organisational resilience capabilities, we adopt the key capabilities required at each phase of the process for organisational resilience, previously proposed by Duchek [6]

\begin{tabular}{|c|c|}
\hline Capability & Phase \\
\hline Observation & Anticipation \\
\hline Identification & Anticipation \\
\hline Accepting & Coping \\
\hline Reflection & Adaptation \\
\hline Learning & Adaptation \\
\hline
\end{tabular}

Table 2. Capabilities at each phase of the OR [6]

We recognise that these capabilities, discussed in the OR literature do not consider BA capabilities. Thus, we see the need for their reconceptualization to include opportunities created by BA. For example, the observation capability, as defined by [6], could be expanded to include monitoring and collection of data related to the so-called 'weak signals' in the business environment. The identification capability requires data on disruptive events, while the accepting capability require data to support scenarioplanning and impact analysis. Reflection and learning, again require data on action taken to 
articulate lessons learned and plan for the future. In addition to providing data, BA systems empower decision-makers to gain insights and make situational decisions across all stages of the OR process. We posit that BA-enabled decision making could therefore be used to enhance the capabilities of observation, identification, accepting, reflection and learning.

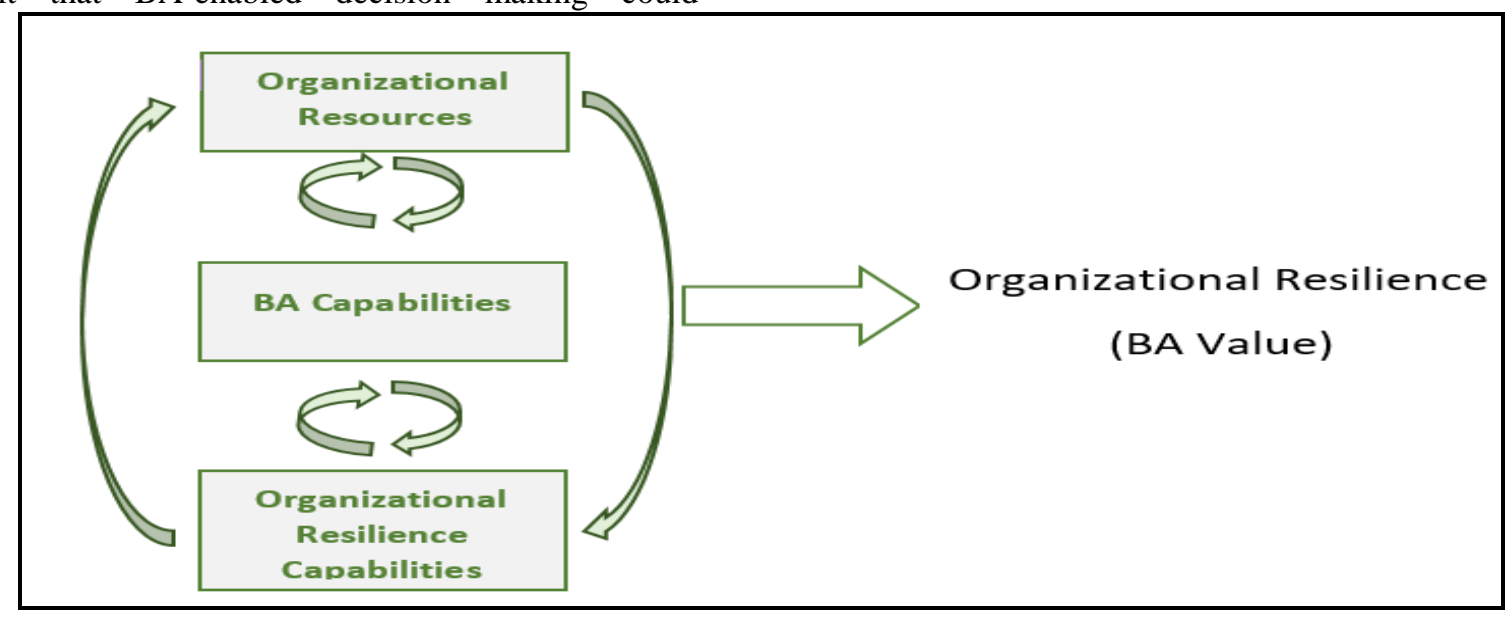

Figure 3. - A proposed conceptual framework of BA capabilities for OR

Taken together, the three components of our proposed framework - Organisational resources, BA capabilities, and Organizational resilience capabilities lead to more sustainable organisational resilience, through mutually-shaping interactions. Based on this framework, we argue that OR could be seen as a novel type of BA value, which is different from the notion of BA Value discussed in the mainstream BA literature [32, 34-36]. Here we see an important opportunity to expand the current research on BA value creation.

\section{6. - Conclusions, Limitations and Future research directions}

Demands and pressure for organization to be resilient cannot be higher in the face of COVID-19 pandemic. This research highlights an important gap within current BA research, as it is yet to focus on OR. We used the literature review to explore and confirm this gap, leading to the proposed conceptualization a conceptual framework of BA capabilities for OR.

The proposed research framework, although preliminary in nature, opens a number of interesting research questions, as follows: What organizational resources could contribute to the development of BA capabilities for OR?, Is there a relation between certain group of BA capabilities and specific OR capabilities? What are the mechanisms through which these components lead to improved OR? How to combine BA and OR capabilities in the most effective way in order to improve OR? We envisage that future refinement of the proposed framework will lead to more research questions for both BA and OR researchers.

We also argue that OR is an important and exciting future research direction for BA researchers, interested in exploring BA capabilities beyond business value creation. Our research findings confirm that this line of research is very much needed, and we argue urgent.

Although we aimed to provide a comprehensive review of both areas of OR and BA capabilities, our literature review is still be limited to only research publications as found through our described approach. Yet, given our intended research contribution to BA, this literature review, was still appropriate to gain an understanding of BA in the mainstream OR research. The dynamic nature of both OR and BA capabilities areas also contributes to the limitation of the research.

Our current research focuses on a particular industry sector (Cooperatives and Mutuals - CMEs) and includes empirical research of BA capabilities used by cooperatives and mutual organizations to enable more sustainable OR. We also hope that the research presented in this paper will open up a new research direction for BA researchers, interested in contributing to this critically important topic of OR.

\section{References}

1. Global, P.s., Crisis Preparedness as the next competitiveadvantage: Learning from 4,500 
crises, PwC's Global Crisis Survey 2019. PwC's Global Crisis Survey 2019, 2019.

2. The Global Risks Report 2019 14th Edition Insight Report. 2019, World Economic Forum: Geneva.

3. Martin Hirt, K.L., and Mihir Mysore Bubbles pop, downturns stop. 2019.

4. Heeks, R. and A.V. Ospina, Conceptualising the link between information systems and resilience: A developing country field study. Information Systems Journal, 2019. 29(1): p. 70-96.

5. Williams, T.A., et al., Organizational response to adversity: Fusing crisis management and resilience research streams. Academy of Management Annals, 2017. 11(2): p. 733-769.

6. Duchek, S., Organizational resilience: a capability-based conceptualization. Business Research, 2019.

7. Hillmann, J., et al., Educating Future Managers for Developing Resilient Organizations: The Role of Scenario Planning. Journal of Management Education, 2018. 42(4): p. 461-495.

8. The Future of Trust: Research Event Recap. 2019, Filene.

9. Edward Barriball, K.G., Ignacio Marcos, and Philipp Radtke, Jump-starting resilient and reimagined operations, in Mckinsey Quarterly. 2020, Mckinsey.

10. Mikalef, P., et al., Big Data Analytics Capabilities and Innovation: The Mediating Role of Dynamic Capabilities and Moderating Effect of the Environment. British Journal of Management, 2019. 30(2): p. 272-298.

11. Samba, C., et al. Organizational Resilience and Positive Leadership: An Integrative Framework. in Academy of Management Proceedings. 2017. Academy of Management Briarcliff Manor, NY 10510.

12. Donnellan, B., T.J. Larsen, and L. Levine, Editorial introduction to the special issue on: transfer and diffusion of IT for organizational resilience. Journal of Information Technology, 2007. 22(1): p. 3-4.

13. Catalan, C. and B. Robert. Evaluation of organizacional resilience: application in Quebec.

14. Burnard, K. and R. Bhamra, Organisational resilience: development of a conceptual framework for organisational responses. International Journal of Production Research, 2011. 49(18): p. 5581-5599.

15. Duchek, S., S. Raetze, and I. Scheuch, The role of diversity in organizational resilience: a theoretical framework. Business Research, 2019: p. 1-37.

16. Bhamra, R., S. Dani, and K. Burnard, Resilience: the concept, a literature review and future directions. International Journal of Production Research, 2011. 49(18): p. 5375-5393.

17. Gupta, M. and J.F. George, Toward the development of a big data analytics capability.
Information \& Management, 2016. 53(8): p. 1049-1064.

18. Chen, H., R.H.L. Chiang, and V.C. Storey, Business intelligence and analytics: from big data to big impact. MIS Q., 2012. 36(4): p. 11651188.

19. Watson, H.J., Tutorial: Big data analytics: Concepts, technologies, and applications. Communications of the Association for Information Systems, 2014. 34(1): p. 65.

20. Torres, R., A. Sidorova, and M.C. Jones, Enabling firm performance through business intelligence and analytics: $A$ dynamic capabilities perspective. Information \& Management, 2018. 55(7): p. 822-839.

21. Wamba, S.F., et al., Big data analytics and firm performance: Effects of dynamic capabilities. Journal of Business Research, 2017. 70: p. 356365 .

22. Marjanovic, O. and B. Dinter, Learning from the History of Business Intelligence and Analytics Research at HICSS: A Semantic Text-mining Approach. Communications of the Association for Information Systems, 2018. 43(1): p. 40.

23. Marjanovic, O., B. Dinter, and T. R Ariyachandra, Introduction to the Minitrack on Organizational Issues of Business Intelligence, Business Analytics and Big Data. 2018.

24. Aral, S. and P. Weill, IT Assets, Organizational Capabilities, and Firm Performance: How Resource Allocations and Organizational Differences Explain Performance Variation. Organization Science, 2007. 18(5): p. 763-780.

25. Wixom, B.H., B. Yen, and M. Relich, Maximizing Value from Business Analytics. MIS Quarterly Executive, 2013. 12(2).

26. Asadi Someh, I. and G. Shanks, How business analytics systems provide benefits and contribute to firm performance? 2015.

27. Webster, J. and R.T. Watson, Analyzing the past to prepare for the future: Writing a literature review. MIS quarterly, 2002: p. xiii-xxiii.

28. Vom Brocke, J., et al. Reconstructing the giant: on the importance of rigour in documenting the literature search process. in Ecis. 2009.

29. Linnenluecke, M.K., Resilience in Business and Management Research: A Review of Influential Publications and a Research Agenda. International Journal of Management Reviews, 2017. 19(1): p. 4-30.

30. Bhamra, R., Organisational resilience: concepts, integration, and practice. 2015: CRC Press.

31. Sincorá, L.A., et al., Business analytics leveraging resilience in organizational processes. RAUSP Management Journal, 2018. 53(3): p. 385-403.

32. Mikalef, P., et al., Big data and business analytics: A research agenda for realizing business value. Information \& Management, 2020. 57(1): p. 103237. 
33. Cosic, R., G. Shanks, and S.B. Maynard, A business analytics capability framework. Australasian Journal of Information Systems, 2015. 19.

34. Shanks, G. and R. Sharma, Creating value from business analytics systems: the impact of strategy. 2011.

35. Günther, W.A., et al., Debating big data: A literature review on realizing value from big data. The Journal of Strategic Information Systems, 2017. 26(3): p. 191-209.

36. Grover, V., et al., Creating Strategic Business Value from Big Data Analytics: A Research Framework. Journal of Management Information Systems, 2018. 35(2): p. 388-423. 